\title{
Student Behaviour and Performance in Relation to Interaction with On- line Activities in a Postsecondary Music Course
}

\author{
Patricia Boechler, Erik deJong, Mary Ingraham, Luis Fernando Marin \\ University of Alberta, Canada
}

\begin{abstract}
This study is an extension of previous research on the infusion of technology into a postsecondary music course to promote the skill of close-listening of music. Due to many in-class hindrances (e.g., time, equipment, acoustics, class size) students in postsecondary music courses do not often experience quality listening opportunities to be able to detect important musical elements. For this study, we developed on-line, supplemental listening activities using Articulate Storyline, Adobe Connect and the virtual world OpenSim. We pretested students on music experience, computer experience and level of self-regulation. At the end of the course, students answered a survey on their enjoyment, tendency to recommend, engagement, perceived increase in understanding of material and whether the activities were worthwhile. In a comparison of 2014 and 2015 results, we found that students with high selfregulation levels rated the above items more positively when the content included more advanced musical concepts. In addition, we found that students who interacted with the supplemental on-line activities and materials achieved higher grades than those who did not. Students who accessed the comprehensive on-line activities close to when the material was presented in class performed better than those who waited until exam time.
\end{abstract}

\section{Introduction}

Due to many in-class hindrances (e.g., time, equipment, acoustics, class size) students in postsecondary music courses do not often experience enough quality listening opportunities to be able to detect various important musical elements [1]. To attend to these difficulties, we have developed a series of technology-based supplemental asynchronous listening activities that are implemented on-line, outside of the classroom. According to Akilli [2], one of the most substantive obstacles to the optimal integration of technology into educational activities is the lack of a guiding development model that is based on learning theory and supporting research. To address this problem, we took great care in developing a theoretical framework to guide the development of our technology-based educational activities, specifically targeted at music content [3]. This was accomplished in two steps: 1) Understanding the cognitive processes involved in the close listening of music, and 2) employing a knowledge acquisition theory that aligned closely to those cognitive processes.

For step one, we relied on Honing's description of the cognition of music [4]. His theory is based on the premise that all humans are born with the innate ability to distinguish different musical elements but through the process of learning our own cultural music conventions this skill becomes implicit, that is, not available to our conscious awareness. Therefore, what is needed is an approach that moves this implicit skill towards conscious awareness, making it explicit and accessible. For step two, we looked to Karmiloff-Smith's Representational Redescription (RR) Model [5] which explains, through a series of four stages, how knowledge moves from implicit to explicit.

After combining these two theories to create our guiding framework, each technology was chosen to increasingly move the learners' cognitive processes from implicit to explicit allowing for communication and sharing of their understanding of the musical concepts. Our first on-line activity used interactive presentations authored with Articulate Storyline to allow the students to listen to pairs of music and make judgements about the musical elements they hear. This independent and self-paced listening experience is our first step in the RR Model, where the students' innate ability to hear different musical elements becomes available to their conscious awareness through repetitive listening (see Figure 1).

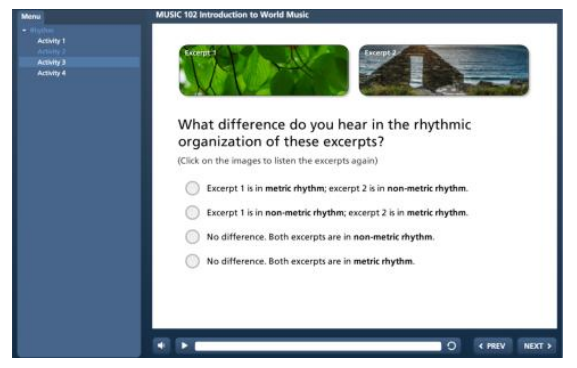

Figure 1. Screenshot from the ARTICULATE Listening Activities

The second listening activity made use of Adobe Connect web-conferencing software for instructor 
facilitated discussions. In these sessions, students use the labels for musical elements they learn from the Articulate sessions and begin expressing their understandings of the elements and their connections to the socio-political context from which the musical examples emerged (see Figure 2).

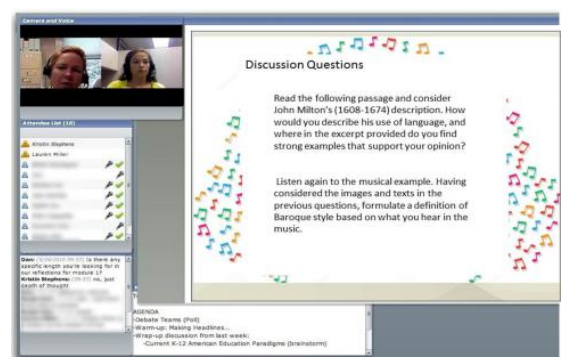

Figure 2. Screenshot from the ADOBE CONNECT Discussion Activities

The last technology, the virtual world simulator OpenSim, allows for a series of peer-based discussions in an immersive environment where students, as avatars, experience cultural aspects and artefacts in multiple forms (e.g. video, audio, historical images and documents, musical scores) from the place and timeframe from which the music originated. These sessions stimulate further linguistic competency in using musical terms while students discuss their knowledge and interpretations with others (see Figure 3).

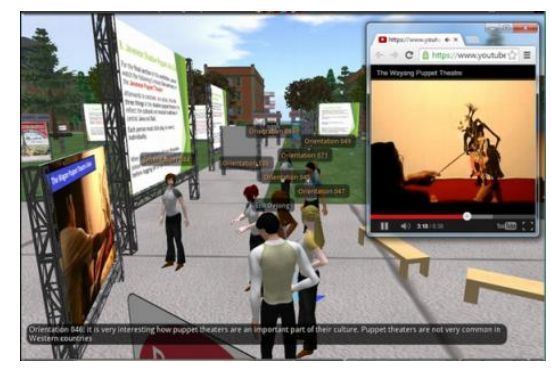

Figure 3. Screenshot from the OPENSIM 3D Activities

\subsection{Advanced Music Content}

Materials incorporated into this project were diverse and represented a complex multimodal environment for student investigation. The goal of the project was to build an understanding of the ways in which the complexity of cultural environments are expressed in music by exposing students to a variety of texts, songs, genres, or instruments from multiple cultures and asking them to listen for distinguishing features. In order to identify such features, students must be able to hear highly nuanced differences in musical sounds and styles.

In preparing supplemental materials for these classes, we considered both basic and advanced content: the fundamentals of music such as rhythm and meter, modality (scales), texture (number of voices or lines of music), and form (organization) are considered as basic content, while advanced content includes more conceptual considerations such as timbre (quality of sound) and the complex expressions of multiple cultures heard within a single piece of music. Both levels of content were presented in the same manner and across all studies, but we incorporated different learning objects within each study to encourage listening for specific features. In the Articulate listening activities, for example, we included the concepts of rhythm and meter as well as timbre and multiculturalism.

Recognizing meter is a fundamental skill in music education and refers to the ability to hear patterns of stressed (strong) and nonstressed (weak) beats; nonmetrical music is described as not having a regular underlying pattern of strong and weak beats. For first-year music students, hearing the difference between metric and nonmetric organization is the primary goal; in advanced listening activities, students are required to listen for groupings of strong and weak beats, and, further, to identify whether the meter is grouped into duple (multiples of two) or triple (multiples of three) meter.

Timbre, or tone colour, defines the quality of a musical sound, and is considered to be a more advanced concept. The ability to recognize timbral difference requires students to hear sometimes nuanced sounds produced by different musical instruments. Such distinctions result from both the material from which the instrument is made as well as the manner in which the sound is produced and whether electronic enhancements are evident. While most students can (and do) hear general timbral difference, they often cannot identify the actual instrument or articulate how or why this difference is produced.

Identifying multiculturalism in music is also complex in that it requires students to listen to individual features (both basic and advanced concepts) and to hear and comprehend their combination within a single piece of music. Here we asked students to listen to and compare musical examples in which a single culture was sounded (monocultural) with those in which more than one cultural tradition (multicultural) was expressed. Without specific contextual information, students were not required to identify which cultures were heard, but rather to listen for the timbral, rhythmic, melodic, and other distinctions that could be heard as expressing one or more cultures.

Adobe Connect and OpenSim were used to present multiple cultural objects to students for discussion and comparison and to engage them in considering textual, audio, and visual sources across two different platforms. In both, students were given materials to consider on their own, to respond to 
questions relating to each object, and to compare two or more of these objects in formulating an understanding of how musical works fit within a specific cultural context. Historical and traditional forms of expression were compared with newer ones to assist students in understanding the migration of cultural expression across time and place and to situate these within a Canadian context.

\subsection{Self-regulation Skills}

Self-regulation refers to the capacity for selfguidance, the management of voluntary action [6], to the self-generated thoughts, feelings, and actions that are planned and cyclically adapted to attain personal goals [7] [8] [9]. Academic self-regulation refers to students' abilities to be proactive about their own learning, through their choices of motivational selftalk, strategies, learning environments and behaviours that move them toward their academic goals. Higher levels of self-regulation have been linked to higher academic achievement [10].

Self-regulated learners are reflective about their learning processes and environments, and monitor the effectiveness of their own thinking and strategies, including the types and degrees of instruction that they need [11], their active approach to learning results in activities, thoughts and feelings that help to create connections between current information and prior learning [12].

In the context of on-line learning, even stronger self-regulation skills are required to maintain motivation and engagement, and to direct effort and planning [13]. In on-line learning, although significant differences in grades of high and low selfregulators may be inconsistent, Artino and Stephens [14] report that low self-regulators experience less cognitive and reflective engagement as well as less satisfaction and willingness to participate in subsequent on-line experiences. Therefore, selfregulation measures were included in both the 2014 and 2015 studies.

Table 1. Supplemental On-line activities offered across 2014 and in 2015

\begin{tabular}{llcccc} 
Course & Enrolled & $\begin{array}{c}\text { Sample } \\
\text { Size }\end{array}$ & Articulate & OpenSim & $\begin{array}{c}\text { Adobe } \\
\text { Connect }\end{array}$ \\
\hline 2014 A & $\mathrm{N}=91$ & $\mathrm{n}=57$ & yes & yes & no \\
\hline $2014 \mathrm{~B}$ & $\mathrm{~N}=44$ & $\mathrm{n}=29$ & yes & no & no \\
\hline 2014 & $\mathrm{~N}=135$ & $\mathrm{n}=86$ & & & \\
\hline 2015 & $\mathrm{~N}=16$ & $\mathrm{n}=13$ & yes & yes & yes \\
\hline Total & $\mathrm{N}=151$ & $\mathrm{n}=99$ & & & \\
\hline
\end{tabular}

The implementation of our supplemental on-line activities was staged across three different courses and we have examined the data in a variety of ways. To make clear which activities were implemented in each course, see Table 1. Given that the two 2014 courses had comparable activities these two samples were aggregated into one dataset for some of our analyses. Given the small sample and difference in activities, 2015 data is handled separately in this report but is included as a potential indicator for future study.

\subsection{Research Questions}

We examined the data from two perspectives. First, we looked at differences between students with varying self-regulation levels and their perceptions of the supplemental on-line activities (2014 and 2015 data) and asked the following questions:

1) Is there a difference between students with high, moderate or low self-regulation and their perceptions of the supplemental on-line activities?

2) Is there an interaction between students with high, moderate or low self-regulation, their perceptions of the supplemental on-line activities and the degree of difficulty of the content (Basic vs. Advanced)?

Second, for the 2014-A data, we looked at relationships and differences between the students' on-line behaviours and their success in the course. For this set of questions, we were interested in students' on-line access to: 1) Comprehensive online activities and, 2) the Supplemental on-line activities only. Comprehensive on-line activities included all available on-line activities and materials, that is, the Supplemental on-line activities along with regular course materials such as readings, course schedules, and assignments. To examine student behaviours from this perspective, we asked:

3) Is there a relationship between students' Comprehensive on-line interactions and each of the performance indicators of Overall grade, the Final Exam grade and the Midterm Exam grade?

4) Is there a difference in performance indicators between students who exhibit high, moderate or low interaction with the Supplemental on-line activities? 5) Were students' access patterns across the course (Comprehensive) correlated with the three performance indicators noted in question 3 ?

\section{Methods}

In each study, the students completed several surveys before starting the listening activities. General demographic information was collected (e.g., program, year in program, gender, and age) as well as: 1) a music experience survey [3], 2) a SelfRegulation Questionnaire (SRQ) [15], and 3) a Computer Experience Questionnaire [16].

The music experience survey asked about the music courses students may have taken and activities 
they typically engaged in outside of their coursework.

The Self-Regulation Questionnaire asked students to rate statements on a 5-point Likert scale. Both positive and negative statements are included in the 63-item questionnaire such as "I usually keep track of my progress toward my goals" and "I get easily distracted from my plans".

The Computer Experience Questionnaire has three parts: 1) the Software Recognition Test (SRT), a measure of general exposure to computer applications and digital materials, 2) the Educational Activities Checklist (EAC), the number of educationrelated computer activities students have carried out (e.g., writing html code, using a formula in a spreadsheet, using a library database), and 3) the Recreational Experience Scale (RES), the number of hours per week students spent playing video games or social networking in Elementary, Junior High, High School and University.

After all on-line activities were completed, students filled out a survey of student perception of value based on a 5-point Likert scale (e.g., "I enjoyed...", "I would recommend...", "I would engage in more of these activities...", "These activities helped my understanding...", "These were worthwhile activities").

Post hoc we also analyzed students' interactions with their course resources and activities on eClass, UofA's Moodle based Learning Management System (LMS). Based on these logs we computed: presence or absence of interaction, proportion of available resources accessed, time of interaction relative to important course milestones, and frequency (number) of interactions. Performance indicators (grades) were also collected and standardized.

\section{Results}

\subsection{Student Perceptions}

For the analyses examining students' perceptions of the Supplemental on-line activities, we used Analysis of Variance to detect differences between students with Low, Moderate or High self-regulation. Below are the results for questions 1 and 2 .

Question \#1. Is there a difference between students with Low, Moderate or High self-regulation and their perceptions of the Supplemental on-line activities?

For the 2014 sample $(n=86$ students in first-year undergraduate general music history courses, groups A \& B), SRQ scores were categorized into high, moderate and low self- regulation skills with a categorical analysis using +/- one standard deviations $(\mathrm{SD}=13.787)$ from their group mean $(\mathrm{M}=213.95)$ as cut off points. We found that, as far as students' perceptions of the listening activities, there was a significant difference across different levels of selfregulatory capacity: students more skilled in regulating themselves perceived less value in highly structured activities (see Figures 4a, 4b). Using a Likert scale where 1 equalled total disagreement and 5 total agreement, students with higher selfregulation on average disagreed that they enjoyed, would recommend, or would engage in more listening activities $[\mathrm{M}=2.77, \mathrm{SD}=.928 ; \mathrm{F}(2,63)=$ $3.877, \mathrm{p}=0.026<.05]$; that these activities had helped them improve their levels of understanding $[\mathrm{M}=2.5, \mathrm{SD}=1.41 ; \mathrm{F}(2,63)=3.613, \mathrm{p}=.033<.05]$ or that these were worthwhile activities in the course $[\mathrm{M}=2.25, \mathrm{SD}=1.28 ; \mathrm{F}(2,63)=5.539, \mathrm{p}=0.006<$ $.05]$.

Question \#2. Is there an interaction between students with Low, Moderate or High self-regulation, their perceptions of the Supplemental on-line activities and the degree of difficulty of the content (Basic vs. Advanced)?

For 2015, again we used Articulate listening activities and OpenSim Virtual Environment discussions but we also introduced an Adobe Connect Web-conferencing discussion which was instructor facilitated and presented students with a variety of musical materials that would enhance their listening and critical thinking skills. This 2015 class, a third-year undergraduate music history course, was significantly different in size $(\mathrm{N}=16)$ and composition from the 2014 dataset. On average participants were further along in years of their programs $[\mathrm{M}=4, \mathrm{SD}=.81 ; \mathrm{F}(1,97)=34.421, \mathrm{p}=$ $.000<.05]$, and more experienced in music $[\mathrm{M}=$ 7.23, $\mathrm{SD}=1.878 ; \mathrm{F}(1,97)=17.288, \mathrm{p}=.000<.05]$, software recognition $[\mathrm{M}=10.77, \mathrm{SD}=3.219 ; \mathrm{F}(1$, $97)=15.225, \mathrm{p}=.000<.05]$ and self-regulatory skills $[\mathrm{M}=223.69, \mathrm{SD}=14.494 ; \mathrm{F}(1,97)=6.201, \mathrm{p}$ $=.014<.05]$. Again, based on their SRQ scores we classified our 2015 sample of students $(n=13)$ into three categories (low, middle and high) using +/- one standard deviations $(\mathrm{SD}=14.494)$ from their group mean $(M=223.69)$ as cut off points.

Since students who were more skilled in regulating themselves in our first sample had perceived less value in these highly structured activities, we would have expected similar or even lower results if we had used the same Listening Activities with the basic musical concepts of rhythm and meter. After all, the students in our second sample were significantly more experienced and had higher levels of selfregulatory ability. To put it in context, if we had used our first sample's cut off points to categorize our second sample, our data would have only loaded into middle and high levels of self-regulation. Therefore, we decided to create an additional challenge and increase the content difficulty, using more advanced concepts for the listening activities. 
We now used three activities including 1) again the Basic concepts of rhythm and meter and two new more advanced musical concepts: 2) timbre and 3) multiculturalism. We developed these new activities using the same structure of interaction, feedback and repeated opportunities for practice as the listening activities in the first study.

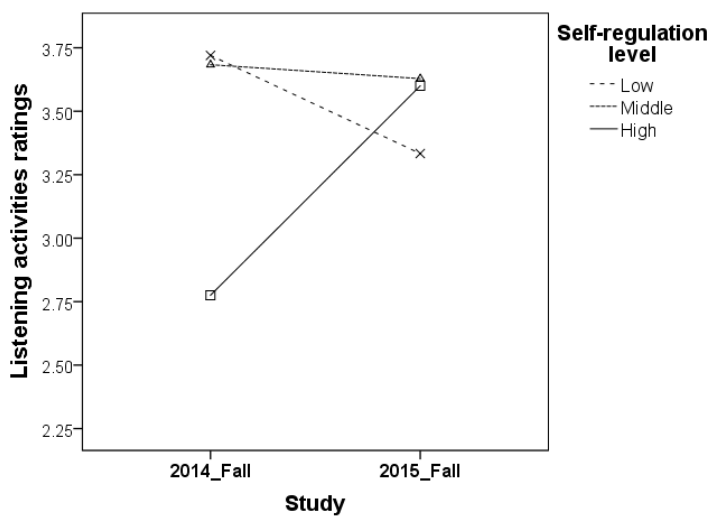

Figure 4. Mean student's rating of on-line listening activities for two different courses and levels of selfregulatory functioning

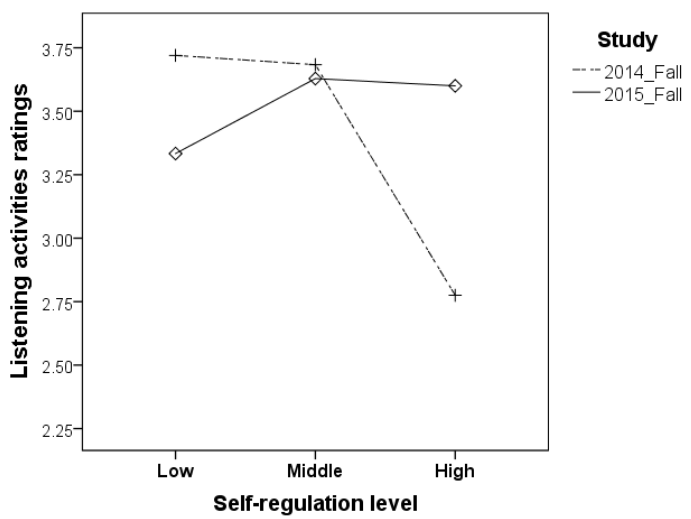

Figure 5. Mean student's rating of on-line listening activities for different levels of self-regulatory functioning and two courses

Our results show that using these more advanced musical concepts removed differences in perceptions between students of different levels of selfregulatory capacity. On average high SRQ students $(\mathrm{SRQ}$ scores $=>\mathrm{M}=223.69+1 \mathrm{SD}=14.494)$ no longer disagreed but enjoyed, would recommend, or would engage in more listening activities $[\mathrm{M}=3.6$, $\mathrm{SD}=.282 ; \mathrm{F}(2,9) 0.335, \mathrm{p}=0.724>.05]$ (see Figures $4 \mathrm{a}$ and $4 \mathrm{~b}$ ); agreed that these activities helped them improve their levels of understanding $[\mathrm{M}=3.5, \mathrm{SD}=.707 ; \mathrm{F}(2,9)=0.110, \mathrm{p}=0.897>.05]$ (see Figure 5) and that these were worthwhile activities in the course $[\mathrm{M}=3.5, \mathrm{SD}=.707 ; \mathrm{F}(2,9)=$ $0.124, \mathrm{p}=0.885>.05]$ (see Figure 6).

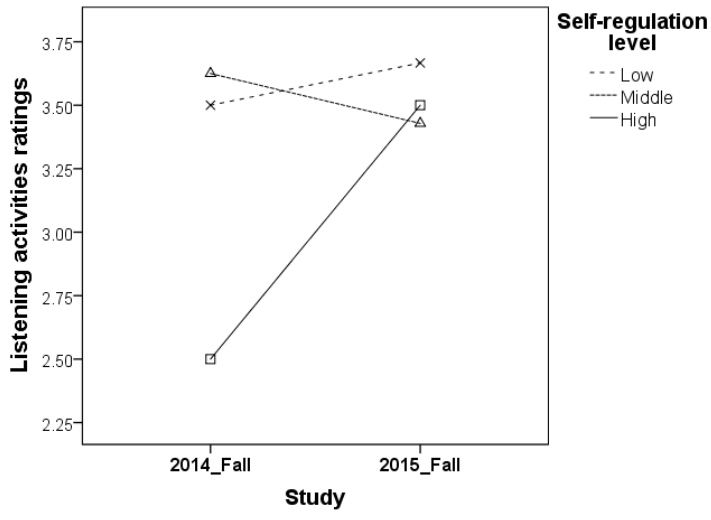

Figure 6. Mean students' ratings of on-line listening activities for the survey item 'The listening activities helped me improve my understanding'

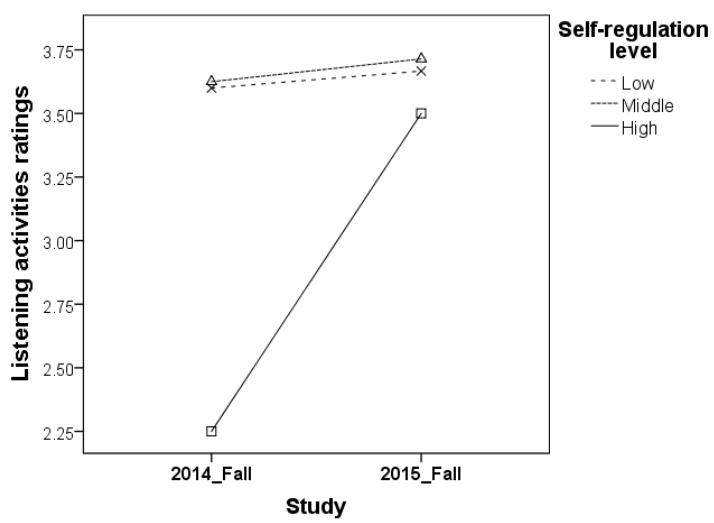

Figure 7. Mean students' ratings of on-line listening activities for the survey item 'The listening activities were worthwhile'

\subsection{Student Behaviours and Performance}

We used Analysis of Variance and correlation analyses on our 2014-A students' behaviours during the course to address the following questions:

Question \#3. Is there a relationship between students' Comprehensive on-line interactions and each of the performance indicators of Overall grade, Final Exam grade and Midterm Exam grade?

The results showed a positive correlation only between students' interactions with the two on-line Supplemental activities and the three performance indicators except for the relationship between students' interactions with OpenSim and the Midterm Exam which was not significant (see Table 2). This is attributable to the timing of this on-line activity in relation to class exam schedules. There was no significant correlation between students' grades and their interactions with any or all other class resources or activities. 
Table 2. Correlations between Articulate and OpenSim access and course grade, final exam and midterm exam grades

\begin{tabular}{lllll} 
& & $\begin{array}{l}\text { Course } \\
\text { Grade }\end{array}$ & $\begin{array}{l}\text { Final } \\
\text { Exam }\end{array}$ & $\begin{array}{l}\text { Midterm } \\
\text { Exam }\end{array}$ \\
\hline \multirow{2}{*}{ Articulate } & Pearson r & $.338^{* *}$ & $.336^{* *}$ & $.340^{* *}$ \\
& Sig. & .001 & .001 & .001 \\
\hline \multirow{2}{*}{ OpenSim } & Pearson r & $.258^{* *}$ & $.280^{* *}$ & .179 \\
& Sig. & .014 & .007 & .094 \\
\hline
\end{tabular}

** Correlation is significant at the .01 level (2-tailed)

Question \#4. Is there a difference in performance indicators between students who exhibit high, moderate or low interaction with the Supplemental on-line activities?

To answer, using K-Means Clustering, we identified three student profiles in our 2014-A sample $(\mathrm{N}=91)$ that represent different levels of interaction with the Supplemental on-line activities. The first profile (Low/Low) represented those students who had low levels of interaction with both Articulate and OpenSim. The second (High/Low) represented students who had high levels of interaction with Articulate and low levels with OpenSim. The third reflected students who had high levels of interaction with both activities (High/High).

We conducted a one-way ANOVA (Profiles X Course, Final Exam, and Midterm Exam Grades) to detect differences between these three student profiles. The analysis revealed a significant difference between the profiles for each performance indicator (Course, $\mathrm{F}(2,87)=6.22, \mathrm{p}=.003)$ (Final Exam, $\mathrm{F}(2,87)=5.73, \mathrm{p}=.005)$ (Midterm Exam, $\mathrm{F}$ $(2,86)=4.28, \mathrm{p}=.017)($ see Table 3 and Figure 7$)$.

Table 3. Mean and standard deviation of standardized course, final exam, and midterm exam grades for three levels of interaction with Articulate and OpenSim supplemental on-line activities

\begin{tabular}{lccc}
\multicolumn{1}{c}{ Mean / SD } & Low-Low & High-Low & High-High \\
\hline Course & $-.488 * / .81$ & $-.022 / 1.0$ & $.385 * / .87$ \\
Final & $-.492 * / .97$ & $-.003 / .99$ & $.355 * / .88$ \\
Midterm & $-.464 * / .91$ & $.103 / 1.0$ & $.261 * / .90$
\end{tabular}

* The mean difference is significant at the .05 level in each row

Post hoc tests (Bonferroni) were conducted to determine which profiles showed significant differences. For each performance indicator, significant differences were found between the Low/Low and the High/High interaction profiles.

Question \#5. Were students' access patterns to the Comprehensive on-line activities across the course correlated with the three performance indicators?

To answer this question, we categorized each student in our 2014-A sample $(\mathrm{N}=91)$ as either predominantly (more than $50 \%$ of the available resources) accessing on-line material for the first time as it was presented through the course schedule or predominantly reviewing on-line material for the first time before exams. Using a one-way ANOVA to compare these two groups, we found that students who accessed course resources and activities (such as instructor slides, assignments, additional resources, exam preparation and instructions, syllabus, Articulate and OpenSim) according to the course schedule of material obtained significantly higher course grades $(\mathrm{M}=.338, \mathrm{SD}=.86, \mathrm{~F}(1,88)=6.62, \mathrm{p}$ $=.012)$ and Final Exam grades $(\mathrm{M}=.297, \mathrm{SD}=.92$, $\mathrm{F}(1,88)=5.16, \mathrm{p}=.026)$ but not Midterm grades $(\mathrm{M}=.20, \mathrm{SD}=.94, \mathrm{~F}(1,88)=2.11, \mathrm{p}=.150)($ see Figure 8).

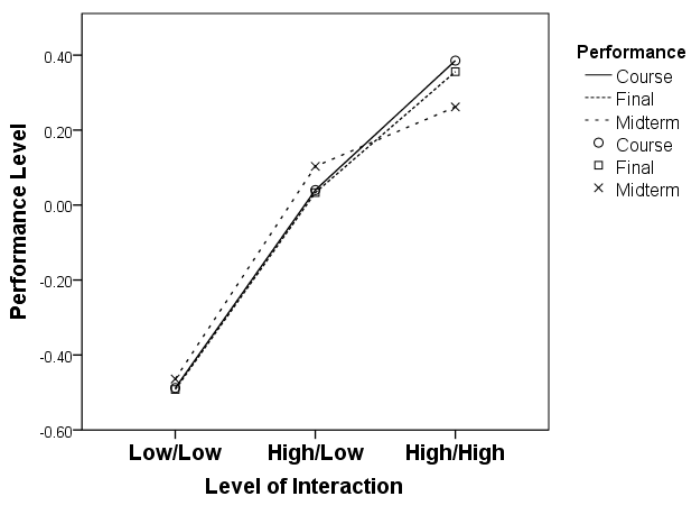

Figure 7. Mean students' standardized grades for three levels of interaction with Articulate and OpenSim supplemental on-line activities

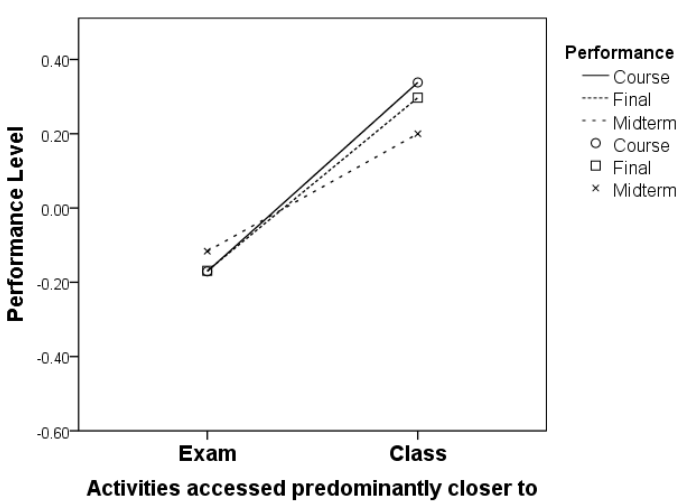

Figure 8. Mean students' standardized grades for two patterns of access to on-line activities

\section{Discussion}

In our first set of questions concerning students' perceptions of the Supplemental on-line activities, we found an interaction between self-regulation skills and the level of complexity of the content. High self-regulators were not as satisfied with or engaged by basic musical content as low and 
intermediate self-regulators but were equally satisfied when advanced content was presented.

We have referred to the overall results of the final student survey as the students' perceived value of the activities. This is comparable to what Eccles and Wigfield [17] refer to as task value, the degree to which students rate an activity as engaging, relevant, and beneficial. In on-line activities specifically, task value has been positively correlated to academic achievement and satisfaction, and the use of cognitive and metacognitive strategies [10]

Given that high self-regulators are strategic and reflective about what activities are more effective for reaching their academic goals, perhaps the Supplemental on-line activities with basic content did not represent a high enough task value to be rated as worthwhile.

Our second set of questions looked at relationships and differences in behaviours using the on-line activities and resulting student performance. We examined both the use of all the on-line activities together (Comprehensive) as well as the Supplemental activities alone and came to the following conclusions.

We found that there is a relationship between students accessing the Supplemental activities and their performance on most of the performance indicators. The student interaction profiles showed that there was a significant difference in performance between the students who had very low interaction and the students who had very high interaction. The exception was the Midterm Exam. Reviewing the timing of events, OpenSim activities occurred after the Midterm Exam, and before the Final Exam. The difference with students with moderate interaction was statistically non-significant.

Across all content in the course, the timing of access to Comprehensive on-line activities played a critical role in performance. When we looked closely at when students accessed the Comprehensive online activities, we found that those who accessed their materials for the first time predominantly when the materials were presented in the course performed better than those who accessed these materials for the first time predominantly before exams. This suggests that the pairing of the Comprehensive activities/materials with the exact materials in the course was a much more effective way of learning the material than cramming before exams.

\section{Conclusions}

Understanding the role of music in cultural contexts is a complex, interdisciplinary activity. In our undergraduate music history curriculum, we lead students through four competencies at all stages of their course work, encouraging them to develop the skills for communicating their understanding of music as a unique form of cultural expression. In our first study (Fall 2014), we focused on cultivating the fundamental competency of listening in first-year university music history students; developing other competencies in reading music, critical thinking and communication were also built into this study, but were largely presented as methods for enhancing listening skills. However, in consideration of the interdisciplinary nature of cultural studies, students interested in music must also be able to 'hear' the impact of visual materials - to interpret their meaning(s) in connection with musical sounds - and to understand connections between written documentation and sounds produced within a specific social and political context. A 2007 study by cognitive psychologist Willingham, suggests that critical thinking must be taught within the context of the discipline in which it is applied in order to be effective in bringing students to higher-level abilities [18]. Our experience in music education classrooms supports this position, and we have set out in these two studies to develop technology-based learning materials that would both encourage critical thinking in understanding music's place in a wider cultural context and that would provide opportunities for students to communicate their discoveries through peer discussion and written communication.

Alongside the challenges of teaching the complex role of music within specific cultural contexts, we need to be aware of the interactions between individual differences in students and the level of complexity of the content that we present. In this instance, advanced content produced different results than basic content in relation to students' selfregulation skills. It is also clear that the timing of access and amount of interaction with on-line materials can lead to improved student performance. Just like any other course materials, on-line materials and activities are best incorporated when students are first exposed to related class material.

\section{References}

[1] P. Boechler, and M. Ingraham, "The Cognition of Listening: A Cognitive Framework for the Blended Delivery of Music Curriculum." In Proceedings of the London International Conference on Education (LICE), Infonomics Society. London, UK, 2014, pp. 119-123.

[2] G. K. Akilli' "Games and Simulations: A New Approach to Education?" In D. Gibson, C. Aldrich \& M. Prensky (Eds.), Games and Simulations in Online Learning: Research and Development Frameworks, Information Science Publishing. Hershey, PA, 2007, pp. 1-20.

[3] P. Boechler, M. Ingraham, L. Fernando Marin, B. Dalen, and E. deJong, "Making the Implicit Explicit: Music Listening, Blended Delivery and the Representational Redescription Model.” In International Journal for Cross-Disciplinary Subjects in Education 
(IJCDSE), 6(1). Infonomics Society, London, UK, 2015, pp. 2095-2105.

[4] H. Honing, Musical Cognition: The Science of Listening. Transaction Publishers, Rutgers, NJ, 2009.

[5] A. Karmiloff-Smith, Beyond modularity: A developmental perspective on cognitive science. MIT Press, Cambridge, MA, 1992.

[6] P. Karoly, "Mechanisms of self-regulation: A systems view." In L. W. Porter \& M. R. Rosenzweig (Eds.), Annual review of psychology, 44(1). Wiley Blackwell, Palo Alto, CA, 1993, pp. 23-52.

[7] B. J. Zimmerman, "A social cognitive view of selfregulated academic learning." Journal of educational psychology, 81(3). Francis, Ipswich, MA, 1989, p. 329.

[8] A. Bandura, "Social cognitive theory of selfregulation." Organizational behavior and human decision process, 50(2). Francis, Ipswich, MA, 1991, pp. 248-287.

[9] D. H. Schunk, and P. A. Ertmer, "Self-regulatory processes during computer skill acquisition: Goal and selfevaluative influences." Journal of educational psychology, 91(2). Francis, Ipswich, MA, 1999, p. 251.

[10] D. H. Schunk, and E. L. Usher, "Barry Zimmerman's Theory of Self-Regulated Learning." In H. Bembenutty, T. J. Cleary, \& A. Kitsantas (Eds). Applications of selfregulated learning across diverse disciplines. A tribute to Barry J. Zimmerman. Information Age Publishing, Charlotte, NC, 2013, pp. 1-28

[11] L. Sha, C. Looi, W. Chen, P. Seow, and L. Wong, "Recognizing and measuring self-regulated learning in a mobile learning environment." In Computers in Human Behavior, 28. Science Direct, Ipswich, MA, 2012, pp. 718728.

[12] H. W. Lee, K. Y. Lim, and B. Grabowski, "Generative Learning strategies and metacognitive feedback to facilitate comprehension of complex science topics and self-regulation." In Journal of Educational Multimedia and Hypermedia, 18(1). ERIC, Ipswich, MA, 2009, pp. 5-25.

[13] N. Dabbagh, and A. Kitsantas, "Supporting selfregulation in student-centered Web-based learning environments." In International Journal on E-Learning, 3. ERIC, Ipswich, MA, 2004, pp. 40-47.

[14] A. R. Artino, and J. M. Stephens, "Beyond grades in online learning: Adaptive profiles of academic selfregulation among naval academy undergraduates." In Journal of Advanced Academics. ERIC, Ipswich, MA, 2009, pp. 568-601.

[15] J. M. Brown, W. R. Miller, and L. A. Lawendowski, "The Self-Regulation Questionnaire." In L. VandeCreek \& T. L. Jackson (Eds.), Innovations in clinical practice: A source book. Professional Resource Press, Sarasota, FL, 1999, pp. 281-289.

[16] P. Boechler, K. Dragon, and E. Wasniewski, "Relationships between Digital Literacy and Print
Literacy: Predictors of Successful On-line Search.” In P. Resta (Ed.), Proceedings of Society for Information Technology \& Teacher Education International Conference. AACE, Chesapeake, VA, 2012, pp. 17551758 .

[17] J. Eccles \& A. Wigfield, "In the mind of the actor: The structure of adolescents' achievement task values and expectancy-related beliefs." In Personality and Social Psychology Bulletin, 21. Sage Publications, Ipswich, MA, 1995, pp. 215-225.

[18] D.T. Willingham, "Critical Thinking: Why is it so hard to teach?" In American Educator. American Federation of Teachers, Washington, DC, 2007, 8-19. 